\title{
Superresolution Linear Optical Imaging in the Far Field
}

\author{
A. A. Pushkina ${ }^{1, *}$ G. Maltese, ${ }^{1, *}$ J. I. Costa-Filho ${ }^{1,},{ }^{1 *}$ P. Patel $\odot,{ }^{1}$ and A. I. Lvovsky $\oplus^{1,2,3, \dagger}$ \\ ${ }^{1}$ Department of Physics, University of Oxford, Oxford OX1 3PU, United Kingdom \\ ${ }^{2}$ Russian Quantum Center, 100 Novaya Street, Skolkovo, Moscow 143025, Russia \\ ${ }^{3}$ P. N. Lebedev Physics Institute, Leninskiy prospect 53, Moscow 119991, Russia
}

(Received 27 April 2021; revised 3 August 2021; accepted 16 November 2021; published 15 December 2021)

\begin{abstract}
The resolution of optical imaging devices is ultimately limited by the diffraction of light. To circumvent this limit, modern superresolution microscopy techniques employ active interaction with the object by exploiting its optical nonlinearities, nonclassical properties of the illumination beam, or near field probing. Thus, they are not applicable whenever such interaction is not possible, for example, in astronomy or noninvasive biological imaging. Far field, linear optical superresolution techniques based on passive analysis of light coming from the object would cover these gaps. In this Letter, we present the first proof-ofprinciple demonstration of such a technique for $2 \mathrm{D}$ imaging. It works by accessing information about spatial correlations of the image optical field and, hence, about the object itself via measuring projections onto Hermite-Gaussian transverse spatial modes. With a basis of 21 spatial modes in both transverse dimensions, we perform two-dimensional imaging with twofold resolution enhancement beyond the diffraction limit.
\end{abstract}

DOI: 10.1103/PhysRevLett.127.253602

The quest for improving resolution in optical imaging has always stumbled upon a seemingly unbreakable wall: the diffraction limit. The light field from the object, as it propagates through the imaging system, experiences diffraction, which gives rise to the smearing of the image. The diffraction limit is usually defined in terms of the heuristic Rayleigh criterion $\theta=1.22 \lambda / D$, where $\theta$ is the resolvable angular separation, $\lambda$ is the wavelength of light, and $D$ is the diameter of the objective lens' aperture [1,2]. It bounds the resolution of optical microscopes to around $200 \mathrm{~nm}$.

The diffraction limit is valid when objects are illuminated by classical light, the image is acquired in the far field, and the involved imaging processes are linear [3,4]. In the last decades we have witnessed an explosion of the socalled superresolution techniques, which could surpass the diffraction limit by breaking at least one of the aforementioned assumptions. By using, e.g., nonlinear excitation of fluorophores [5,6], utilizing their distinguishability in time [7,8], or near field probing of evanescent waves [9], they were able to get around the diffraction barrier and bring optical microscopy to the nanoscale.

Each of these methods, however, requires direct interaction with the sample and/or certain nonlinear properties thereof and hence comes with a host of limitations, in addition to significant cost and complexity. In certain cases, such as astronomical imaging or microscopy of certain sensitive samples [10], the active nature of the interaction with the object precludes the application of existing superresolution techniques altogether.

A recent breakthrough [11], however, has shown that superresolution can be achieved in the far field, with linear optics, and for standard illumination. It hinges on the discovery that the optical field's spatial correlations, which are ignored in conventional direct intensity measurements, contain additional information about the object. That information can be accessed by coherently processing the image field just before its detection and therefore does not require any active manipulation of the sample.

One way to carry out this coherent processing is spatialmode sorting or demultiplexing [11] of the image field, i.e., decomposing it into a basis of spatial modes, e.g., the Hermite-Gaussian (HG) basis [11,12], and measuring the magnitude of each component. The shape of the object is then reconstructed from these measurements. Our approach is, therefore, reminiscent of the so-called single-pixel imaging $[13,14]$, which utilizes spatial-mode decomposition where array detectors are unavailable because of wavelength restrictions or rapid acquisition requirements.

Intuitively, our approach helps achieving superresolution by leveraging the fine spatial structure of these modes: since the size of their features scales with the inverse square root of the mode order, measuring the image field's projections into higher-order modes accesses increasingly finer details of the spatial distribution of the field correlations and, therefore, the subwavelength information they carry.

The original theoretical idea [11], as well as all existing experimental work [15-20] focus on estimating just one or several parameters of the object, such as the separation between two point sources. Here we report the first experimental demonstration of this approach in application to full two-dimensional imaging. Our work is based on the 
method proposed theoretically by Yang et al. [15] and dubbed Hermite-Gaussian microscopy (HGM). Tsang has shown the advantage of this approach in comparison with direct imaging in terms of quantum Fisher-information formalism $[21,22]$. In practice, we are able to resolve the objects' details at one-half of the diffraction limit imposed by the optical system.

Implementing HGM experimentally faces a number of challenges. First, the image reconstruction method of Ref. [15] assumes an ideal optical system with no aberrations and a perfectly Gaussian point spread function. Second, it requires precise measurement of the spatial mode in the HG basis, which must be perfectly matched to the image field. This requirement is further complicated by the signal magnitudes associated with higher- and lowerorder modes differing by several orders of magnitude. Third, the method of Ref. [15] breaks down in the presence of noise; particularly, it is vulnerable to the shot noise inherent to the quantum nature of light (see Supplemental Material [23]).

We overcome these challenges as follows. Instead of implementing direct HG mode demultiplexing of the image field, we perform sequential heterodyne detection of that field with different $\mathrm{HG}$ beams as local oscillators (LOs). The heterodyne detector is sensitive only to the component of the field that matches the LO mode, effectively selecting the necessary field projections. Since it is technically easier to prepare HG modes [27,28] than sort them [29-31], this approach significantly simplifies the experiment. We acquire heterodyne photocurrents for $\mathrm{HG}_{m, n}$ with $m$ and $n$ ranging from 0 to 20 .

We overcome the imperfections of the imaging system and the systematic errors by using machine learning, rather than a rigid theoretical model, to calibrate our measurements. We train a neural network $(\mathrm{NN})$ to reconstruct a superresolved image from the measurement data by presenting it with these data for a variety of known objects. This training enables the NN to reconstruct an unknown object from a set of measurements acquired with the same setup.

This proof-of-principle experiment is implemented in a simplified setting with coherently illuminated samples and an imaging system with a low numerical aperture. A schematic of the setup is presented in Fig. 1. A continuous laser beam at $785 \mathrm{~nm}$ is initially split into the signal and LO paths. At the signal path, the beam is frequency shifted by 92.05 MHz via an acousto-optic modulator before illuminating a binary amplitude mask (the object to image) displayed via a digital micromirror device (DMD). The objects are generated inside a $210 \times 210$ pixel area, with a pixel pitch of $7.56 \mu \mathrm{m}$. To impose the diffraction limit, the light reflected from the DMD is imaged by an objective lens placed at a distance of $245.5 \mathrm{~cm}$ and with a numerical aperture (NA) reduced to $7.1 \times 10^{-4}$ by an iris of $3.5 \mathrm{~mm}$ diameter placed in front of it. The corresponding

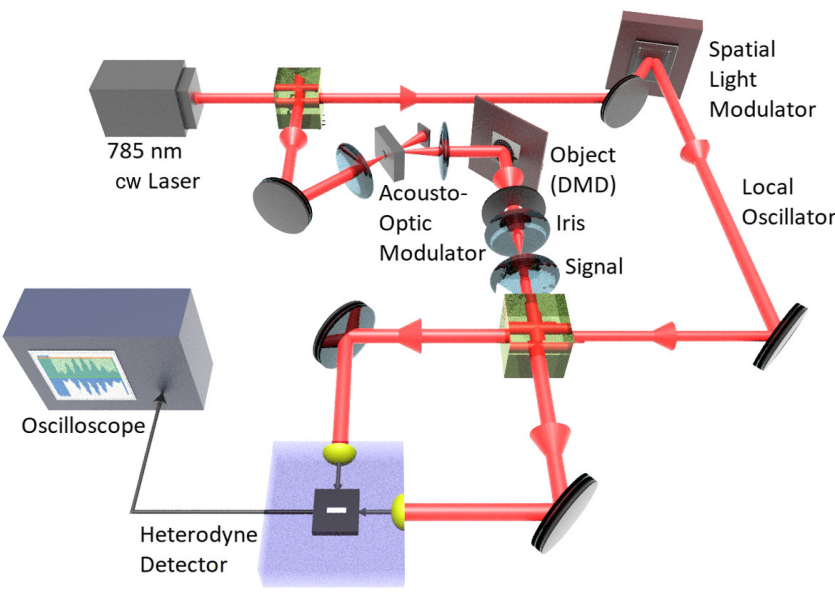

FIG. 1. Schematic of the experiment. The object is a $210 \times$ 210 pixel bitmap displayed on the DMD; the spatial modes of the local oscillator are prepared using a liquid-crystal SLM.

(theoretical) coherent light Rayleigh limit $1.64 \lambda / 2 \mathrm{NA}$ is now $906 \mu \mathrm{m}$ (120 DMD pixels) and, for comparison, the classical incoherent light limit $1.22 \lambda / 2 \mathrm{NA}$ is $674 \mu \mathrm{m}(89$ DMD pixels). In the LO path, the laser beam is shaped into a $\mathrm{HG}_{m, n}$ mode by a liquid-crystal spatial light modulator (SLM). Using the scheme of Ref. [32], which allows independent phase and amplitude modulation of the beam, plus a previously developed procedure [27] to compensate for the SLM's imperfections, we are able to generate highquality HG modes up to the 20th order in both directions. Finally, the signal and LO paths are recombined for heterodyne detection. The produced photocurrents are demodulated; their phases and amplitudes are recorded [23].

The signal-to-noise ratio of the acquired signal was limited by technical noise and amounted, dependent on the object, to 25-35 dB for lowest-order modes and 0-10 dB for highest-order modes. This ratio was typically poorer for odd modes because most objects had a dominant symmetric component, which gave rise to higher signal in even modes [Fig. 2(b)].

The complex photocurrent values associated with the 441 LO modes constitute the experimental data for the image reconstruction. Note that both the amplitudes and phases are needed for the imaging process: without the phases, we are unable to reconstruct the antisymmetric features of the images [15].

The HGM image reconstruction as described in Ref. [15] relies on precise knowledge of the point spread function and is supremely sensitive to even the slightest experimental imperfections. The sources of errors can be manifold: imperfect $\mathrm{HG}$ modes, phase aberrations in both beams' paths, the intrinsic curvature of the DMD surface, hardness and asymmetry of the aperture, and the misalignment between the HG modes and the signal beam, among others. To overcome these issues, we calibrate our imaging system using a supervised feed forward 


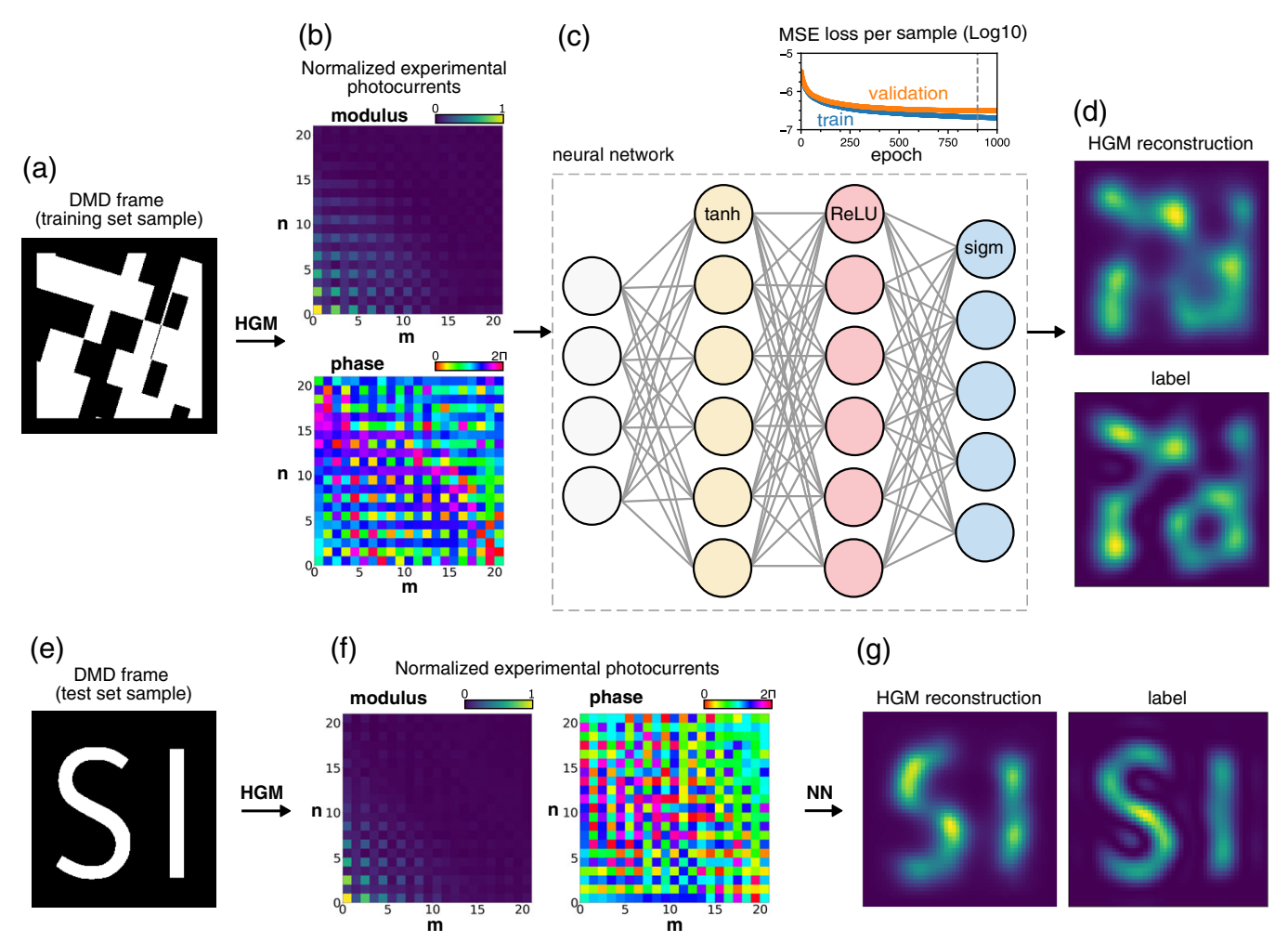

FIG. 2. Sketch of the data acquisition and processing pipeline. We image via HGM a sample (a) and acquire its complex photocurrents (b), which are then fed to the $\mathrm{NN}$ (c) (the inset shows the learning curve, i.e., the training and cross-validation mean squared error (MSE) loss versus the training epoch). The NN is trained to predict the idealized HGM reconstruction of the sample image (d). (e)-(g) The reconstruction of a sample from the test set.

NN [33], schematized in Fig. 2. The input of the network is 441 real and imaginary components of the heterodyne output photocurrents; the output is a $50 \times 50$ bitmap containing the image. The NN architecture is shown in Fig. 2 and contains two hidden layers with 6000 units each.

In order to train the NN, we use the DMD to produce 26 501 training samples consisting of random bitmaps as well as simple geometric shapes [23]. These are divided into the training and cross-validation dataset in the 90:10 proportion. The elements of the training set are sequentially displayed on the DMD, and the corresponding set of complex-valued photocurrents is acquired for each of them.

For the corresponding set of training labels, we do not use the "ground truth" objects (as done, e.g., in Ref. [34]); instead, we simulate the images that would be reconstructed from the training set with the ideal HGM $[15,35]$. With this approach, we train the NN to approximate the underlying HGM model and filter out the experimental noise and not to guess the sample features beyond the resolution capabilities of the optics. We found that using the ground truth objects (or even a slightly smoothed version thereof) as labels leads to overfitting issues that actually degrade the imaging quality for the test set.

After training, we evaluate the NN performance on previously unseen samples: the logo and coat of arms of Oxford University [Figs. 3(a) and 3(b)], pairs of lines of varied separation [Figs. 4(a) and 4(b)] and alphabet characters [Supplemental Material [23], Figs. 7(a) and 7(b)]. The logo and coat of arms have been split into, respectively, 30 and 120 smaller square rasters of size $210 \times 210$ DMD pixels (shown by the grid in Fig. 3), each displayed on the DMD and fed to the NN in sequence. This procedure is equivalent to the transverse scanning of the object with the stride of $210 \times 7.56=1588 \mu \mathrm{m}$.

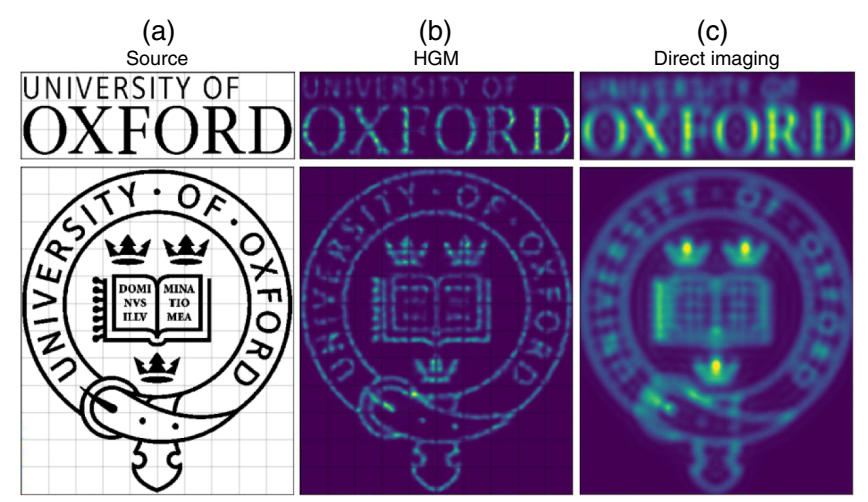

FIG. 3. (a) Original, (b) HGM-reconstructed, and (c) camera images of the Oxford logo and coat-of-arms test sets. The grid shows the division of the images into square subimages that are fed to HGM sequentially. 

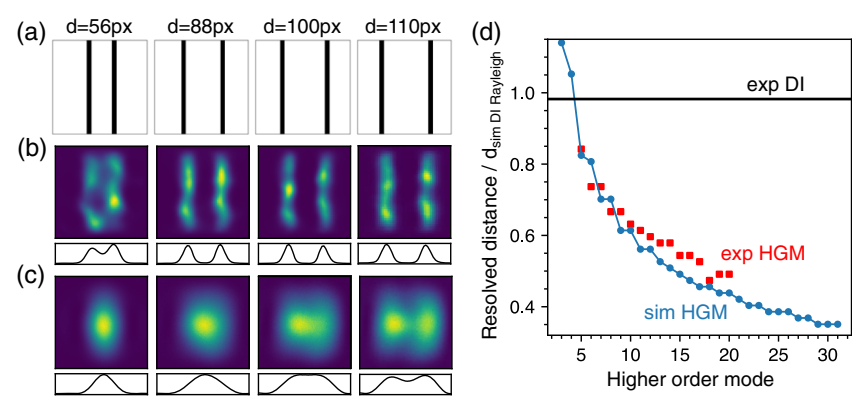

FIG. 4. Qualitative assessment of the resolution improvements of HGM. (a) Lines pairs of increasing spacing, (b) their reconstruction via HGM using $21 \times 21$ modes, and (c) camera images. (d) Rayleigh criterion for experimental (red squares) and simulated (blue connected dots) HGM vs number of used modes, normalized to the value for simulated direct imaging (DI), together with the experimental direct imaging (continuous black line).

In order to compare our performance with traditional direct imaging methods, we perform direct intensity measurements with a camera placed at the image plane of the objective lens [Figs. 3(c) and 4(c); Supplemental Material [23], Fig. 7(c)]. For the logos, the transverse scan has also been simulated as described above, albeit with a smaller stride (10 DMD pixels) because direct imaging was more sensitive to aberrations. Qualitatively, we can see that the HGM reconstructions are much sharper than direct images and allow us to see fine details and features that otherwise could not be distinguished. HGM is also superior to camera images postprocessed with deconvolution algorithms (see Supplemental Material [23]).

In Fig. 4, we quantitatively benchmark the resolution gain by imaging pairs of parallel lines. Examples are shown in Figs. 4(a)-4(c), whereas Fig. 4(d) plots the HGM resolution as a function of the number of HG modes used in both dimensions. To quantify the resolution, the classic Rayleigh criterion is used, i.e., that two sources are considered resolved when the intensity at their midpoint is at most $75 \%$ of the maximum intensity. We find that HGM with up to the $\mathrm{HG}_{20,20}$ mode can resolve two sources at approximately one-half of the diffraction limit. In other words, the HGM resolution is comparable to the direct imaging performed using a lens that is twice as wide.

We can also see from Fig. 4(d) that our experimental results on the resolution are close to the simulations. The HGM resolution is expected to scale approximately as the inverse square root of the number of modes in each dimension. Hence, the theoretically achievable resolution enhancement is significantly higher than that shown here. In practice, an important limitation is associated with generating high-order modes, which is increasingly challenging to do with high fidelity due to the limited SLM resolution.

Superresolution is known to be dramatically degraded by noise [36,37]. The ultimate resolution limit arises from the shot noise, which affects the signal from all modes, but especially high-order HG modes, whose magnitudes rapidly fall with the mode number [38,39]. This is not a limiting factor in our experiment, as the number of photons in each measurement is on a scale of $10^{10}$, corresponding to the signal-to-shot-noise ratio of $50 \mathrm{~dB}$. However, we simulated the shot noise effect in the Supplemental Material [23] and found that, in the presence of that noise, HGM consistently produces higher-quality reconstruction than direct imaging, yielding reasonable reconstruction quality with as few as $10^{3}$ photons per image section.

We now briefly discuss the perspectives of adapting our method for practical imaging, e.g., in microscopy or astronomy. One important difference is that the light sources in practical settings are typically incoherent. In this case, the phases of the heterodyne detector photocurrents are random, but their amplitudes are sufficient to reconstruct the component of the image that is symmetric with respect to the reflection about the horizontal and vertical coordinate axes [15]. The antisymmetric components can then be reconstructed by using superpositions of HG modes as the LO [21] or obviated by shifting the object to a single quadrant of the reference frame [15].

A further limitation of heterodyne detection is the detectable bandwidth, which is bounded by the detector electronics. For practical imaging of broadband objects, one of the spatial-mode demultiplexing methods [20,2931,37] must instead be used.

For the NN training in a microscopic setting, one could rely on off-the-shelf calibration slides and microplates for optical microscopes [40]. A calibration slide of a few tens of micrometer size, containing several thousand training objects of size $0.5-1 \mu \mathrm{m}$, can be fabricated with a resolution of a few tens of nanometers by way of lithography or laser writing. This slide can be scanned in front of the microscope objective in various orientations to increase the straining set size. If the variety and amount of the training data are still not sufficient, one could implement data augmentation techniques [41], adapting them to the HGM NN.

HGM is a vastly simpler and cheaper alternative to many existing superresolution methods. Furthermore, its passive nature permits universal application in a wide variety of imaging scenarios, including those not accessible by existing schemes. HGM can be combined with other imaging techniques to further increase the resolution [42]. This could open up a whole new direction in both the academic and industrial sides of optical imaging.

*These authors contributed equally to this work.

Alex.Lvovsky@physics.ox.ac.uk

[1] L. Rayleigh, XXXI. Investigations in optics, with special reference to the spectroscope, The London, Edinburgh, and Dublin Philos. Mag. J. Sci. 8, 261 (1879). 
[2] G. de Villiers and E. R. Pike, The Limits of Resolution Series in Optics and Optoelectronics (CRC Press, Boca Raton, FL, 2016).

[3] L. Mandel and E. Wolf, Optical Coherence and Quantum Optics (Cambridge University Press, Cambridge, England, 1995).

[4] J. Goodman, Introduction to Fourier Optics (Roberts \& Co, Englewood, CO, 2005).

[5] S. W. Hell and J. Wichmann, Breaking the diffraction resolution limit by stimulated emission: Stimulatedemission-depletion fluorescence microscopy, Opt. Lett. 19, 780 (1994).

[6] S. W. Hell, Far-field optical nanoscopy, Science 316, 1153 (2007).

[7] M. J. Rust, M. Bates, and X. Zhuang, Sub-diffraction-limit imaging by stochastic optical reconstruction microscopy (STORM), Nat. Methods 3, 793 (2006).

[8] R. M. Dickson, A. B. Cubitt, R. Y. Tsien, and W.E. Moerner, On/off blinking and switching behaviour of single molecules of green fluorescent protein, Nature (London) 388, 355 (1997).

[9] U. Drig, D. W. Pohl, and F. Rohner, Near-field opticalscanning microscopy, J. Appl. Phys. 59, 3318 (1986).

[10] M. Tsang, Resolving starlight: A quantum perspective, Contemp. Phys. 60, 279 (2019).

[11] M. Tsang, R. Nair, and X.-M. Lu, Quantum Theory of Superresolution for Two Incoherent Optical Point Sources, Phys. Rev. X 6, 031033 (2016).

[12] J. Rehacek, M. Paúr, B. Stoklasa, Z. Hradil, and L. L. Sánchez-Soto, Optimal measurements for resolution beyond the Rayleigh limit, Opt. Lett. 42, 231 (2017).

[13] M. F. Duarte, M. A. Davenport, D. Takhar, J. N. Laska, T. Sun, K. F. Kelly, and R. G. Baraniuk, Single-pixel imaging via compressive sampling, IEEE Signal Process. Mag. 25, 83 (2008).

[14] M. P. Edgar, G. M. Gibson, and M. J. Padgett, Principles and prospects for single-pixel imaging, Nat. Photonics 13, 13 (2019).

[15] F. Yang, A. Tashchilina, E. S. Moiseev, C. Simon, and A. I. Lvovsky, Far-field linear optical superresolution via heterodyne detection in a higher-order local oscillator mode, Optica 3, 1148 (2016).

[16] Z. S. Tang, K. Durak, and A. Ling, Fault-tolerant and finiteerror localization for point emitters within the diffraction limit, Opt. Express 24, 22004 (2016).

[17] M. Paúr, B. Stoklasa, Z. Hradil, L. L. Sánchez-Soto, and J. Rehacek, Achieving the ultimate optical resolution, Optica 3, 1144 (2016).

[18] W.-K. Tham, H. Ferretti, and A. M. Steinberg, Beating Rayleigh's Curse by Imaging Using Phase Information, Phys. Rev. Lett. 118, 070801 (2017).

[19] M. Parniak, S. Borówka, K. Boroszko, W. Wasilewski, K. Banaszek, and R. Demkowicz-Dobrzański, Beating the Rayleigh Limit Using Two-Photon Interference, Phys. Rev. Lett. 121, 250503 (2018).

[20] P. Boucher, C. Fabre, G. Labroille, and N. Treps, Spatial optical mode demultiplexing as a practical tool for optimal transverse distance estimation, Optica 7, 1621 (2020).
[21] M. Tsang, Subdiffraction incoherent optical imaging via spatial-mode demultiplexing, New J. Phys. 19, 023054 (2017).

[22] M. Tsang, Quantum limit to subdiffraction incoherent optical imaging, Phys. Rev. A 99, 012305 (2019).

[23] See Supplemental Material at http://link.aps.org/supplemental/ 10.1103/PhysRevLett.127.253602 for details of the experiment and shot noise analysis, which includes Refs. [24-26].

[24] R. Kumar, E. Barrios, A. MacRae, E. Cairns, E. Huntington, and A. Lvovsky, Versatile wideband balanced detector for quantum optical homodyne tomography, Opt. Commun. 285, 5259 (2012).

[25] A. Masalov, A. Kuzhamuratov, and A. Lvovsky, Noise spectra in balanced optical detectors based on transimpedance amplifiers, Rev. Sci. Instrum. 88, 113109 (2017).

[26] B. Zhang, J. Zerubia, and J.-C. Olivo-Marin, Gaussian approximations of fluorescence microscope point-spread function models, Appl. Opt. 46, 1819 (2007).

[27] A. A. Pushkina, J. I. Costa-Filho, G. Maltese, and A. I. Lvovsky, Comprehensive model and performance optimization of phase-only spatial light modulators, Meas. Sci. Technol. 31, 125202 (2020).

[28] A. Forbes, A. Dudley, and M. McLaren, Creation and detection of optical modes with spatial light modulators, Adv. Opt. Photonics 8, 200 (2016).

[29] Y. Zhou, J. Zhao, Z. Shi, S. M. H. Rafsanjani, M. Mirhosseini, Z. Zhu, A. E. Willner, and R.W. Boyd, Hermite-Gaussian mode sorter, Opt. Lett. 43, 5263 (2018).

[30] M. Hiekkamäki, S. Prabhakar, and R. Fickler, Near-perfect measuring of full-field transverse-spatial-modes of light, Opt. Express 27, 31456 (2019).

[31] N. K. Fontaine, R. Ryf, H. Chen, D. T. Neilson, K. Kim, and J. Carpenter, Scalable mode sorter supporting 210 HermiteGaussian modes, in Optical Fiber Communication Conference Postdeadline Papers (Optical Society of America, San Diego, 2018), p. Th4B.4.

[32] E. Bolduc, N. Bent, E. Santamato, E. Karimi, and R.W. Boyd, Exact solution to simultaneous intensity and phase encryption with a single phase-only hologram, Opt. Lett. 38, 3546 (2013).

[33] A. M. Palmieri, E. Kovlakov, F. Bianchi, D. Yudin, S. Straupe, J. D. Biamonte, and S. Kulik, Experimental neural network enhanced quantum tomography, npj Quantum Inf. 6, 20 (2020).

[34] W. Ouyang, A. Aristov, M. Lelek, X. Hao, and C. Zimmer, Deep learning massively accelerates super-resolution localization microscopy, Nat. Biotechnol. 36, 460 (2018).

[35] G. Maltese, J. I. Costa-Filho, A. Pushkina, P. Patel, and A. Lvovsky, Hermite-Gaussian Microscopy (GitHub, San Francisco, 2021), https://github.com/giomalt/HGM.

[36] C. Oh, S. Zhou, Y. Wong, and L. Jiang, Quantum Limits of Superresolution in a Noisy Environment, Phys. Rev. Lett. 126, 120502 (2021).

[37] G. Sorelli, M. Gessner, M. Walschaers, and N. Treps, Optimal Observables and Estimators for Practical Superresolution imaging, Phys. Rev. Lett. 127, 123604 (2021).

[38] F. Yang, R. Nair, M. Tsang, C. Simon, and A. I. Lvovsky, Fisher information for far-field linear optical superresolution 
via homodyne or heterodyne detection in a higher-order local oscillator mode, Phys. Rev. A 96, 063829 (2017).

[39] C. Lupo, Subwavelength quantum imaging with noisy detectors, Phys. Rev. A 101, 022323 (2020).

[40] A. D. Corbett, M. Shaw, A. Yacoot, A. Jefferson, L. Schermelleh, T. Wilson, M. Booth, and P. S. Salter, Microscope calibration using laser written fluorescence, Opt. Express 26, 21887 (2018).
[41] C. Shorten and T. M. Khoshgoftaar, A survey on image data augmentation for deep learning, J. Big Data 6, 1 (2019).

[42] K. K. M. Bearne, Y. Zhou, B. Braverman, J. Yang, S. A. Wadood, A. N. Jordan, A. N. Vamivakas, Z. Shi, and R.W. Boyd, Confocal super-resolution microscopy based on a spatial mode sorter, Opt. Express 29, 11784 (2021). 